\title{
ARITHMETIC MEANS OF FOURIER COEFFICIENTS
}

\author{
RAJENDRA SINHA
}

\begin{abstract}
Given the Fourier coefficients of an even continuous function, we find a necessary and sufficient condition such that their arithmetic means are the Fourier coefficients of an odd continuous function. A similar result is shown for those Lipschitz classes whose elements are automatically equivalent to continuous functions.
\end{abstract}

Introduction. Let $\sum_{n=1}^{\infty} a_{n} \cos n x$ and $\sum_{n=1}^{\infty} b_{n} \sin n x$ be the Fourier series of $f_{c}$ and $f_{s}$, respectively. Hardy showed that $\sum_{n=1}^{\infty}\left[\left(a_{1}+\cdots+a_{n}\right) / n\right] \cos n x$ and $\sum_{n=1}^{\infty}\left[\left(b_{1}+\cdots+b_{n}\right) / n\right] \sin n x[2$, p. 51] will also be Fourier series-let $T_{H} f_{c}$ and $T_{H} f_{s}$, respectively, represent these two series. It was shown by $\mathrm{M}$. Kinukawa and S. Igari [5, Theorem 2] that $f_{c} \in L^{\infty} \Rightarrow T_{H} \tilde{f}_{c} \in L^{\infty}$. In this paper we show that if $f_{c} \in C$, then $f_{c}(0)=0 \Leftrightarrow T_{H} \tilde{f}_{c} \in C$.

A. Konyushkov [6, Theorem 15] showed that for $0<\alpha<1 / p \leqslant 1, f_{c} \in \Lambda_{\alpha}^{p}$ $\Rightarrow T_{H} f_{c} \in \Lambda_{\alpha}^{p}$. In this paper we consider the case $0<1 / p<\alpha \leqslant 1$, and show that if $f_{c} \in \Lambda_{\alpha}^{p}$, then $\sum_{n=1}^{\infty} a_{n}=0 \Leftrightarrow T_{H} \tilde{f}_{c} \in \Lambda_{\alpha}^{p}$.

All the notations, unless otherwise mentioned, are taken from [9]. Every function is supposed to be defined a.e., periodic with period $2 \pi$ and integrable on $[-\pi, \pi]$. We shall not distinguish between equivalent functions. Given a function $f$, by $f(t)$ we would mean $\lim _{n \rightarrow \infty} \sigma_{n}(t ; f)$-whenever it exists. For convenience we shall write the Lipschitz classes $\Lambda_{\alpha}^{p}$ and $\Lambda_{\alpha}$ as $\Lambda(\alpha, p)$ and $\Lambda(\alpha)$, respectively.

Definition. For $f$ and $\tilde{f} \in L^{1}$, we define $T_{S} f$ as the function representing the Fourier series $\sum_{n=1}^{\infty}\left[S_{n}(0 ; f) / n\right] \sin n t$.

We can easily check the following:

(i) $T_{S} f_{c}=T_{H} \tilde{f}_{c}$.

(ii) For $0<\alpha \leqslant 1 \leqslant p<\infty$ and $\alpha p \neq 1$,

$$
T_{S} f_{c} \in \Lambda(\alpha, p) \Leftrightarrow T_{H} f_{c} \in \Lambda(\alpha, p) .
$$

(iii) For $0<1 / p<\alpha \leqslant 1$, by [4, Theorem 5 (ii)],

$$
f_{c} \in \Lambda(\alpha, p) \Rightarrow \sum_{n=1}^{\infty} a_{n}=\lim _{n \rightarrow \infty} \sigma_{n}(0 ; f)=f(0) \text {. }
$$

Now we state our results in the following form.

Theorem 1. Let $f \in C$. Then $f(0)=0 \Leftrightarrow T_{S} f \in C$.

Received by the editors January 24, 1975.

AMS (MOS) subject classifications (1970). Primary 42A16; Secondary 26A16.

Key words and phrases. Fourier series, conjugate function, Lipschitz class, strong summability, closed graph theorem. 
TheOREM 2. Let $f \in \Lambda(\alpha, p)$ where $0<1 / p<\alpha \leqslant 1$. Then $f(0)=0$ $\Leftrightarrow T_{S} f \in \Lambda(\alpha, p)$.

Lemma 1.1. For $1<p \leqslant \infty,\left\|T_{S} f\right\|_{p} \leqslant A_{p}\|f\|_{p}$ where $A_{p}$ is a constant depending upon $p$ only.

Proof. Let $f \sim \sum_{n=0}^{\infty}\left(a_{n} \cos n t+b_{n} \sin n t\right)$. Take $f_{c}$ and $f_{s}$ as before. Then

$$
T_{S} f(t)=T_{H} \tilde{f}_{c}(t)+a_{0}(\operatorname{sgn} t)(\pi-|t|) / 2 \text { for a.e. } t \text {. }
$$

Hence, for $1<p<\infty$, by [2, p. 51],

$$
f \in L^{p} \Rightarrow T_{S} f \in L^{p} .
$$

Now using the analysis similar to that of [2], we can show that

$$
T_{H} \tilde{f}_{c}(t)=\int_{t}^{\pi} \frac{\tilde{f}_{c}(u) d u}{2 \tan u / 2}+\beta(t) \text { for a.e. } t>0
$$

where $\sum_{n=1}^{\infty}\left[a_{n} / 2 n\right] \sin n t=\beta(t) \in C$.

Hence, by [6, Theorem 2],

$$
f \in L^{\infty} \Rightarrow f_{c} \in L^{\infty} \Rightarrow T_{H} \tilde{f}_{c} \in L^{\infty} \Rightarrow T_{S} f \in L^{\infty} .
$$

Now using the closed graph theorem, we can easily show the continuity of the operator $T_{S}$.

Proof of Theorem 1. $(\Rightarrow)$ Let $\sigma_{n} f(x) \equiv \sigma_{n}(x ; f)$. Define

$$
e_{n}(x) \equiv \sigma_{n} f(x)-\left[\sigma_{n} f(0)\right] \cos (n+1) x .
$$

Then

$$
\left\|e_{n}-f\right\|_{\infty} \leqslant\left\|\sigma_{n} f-f\right\|_{\infty}+\left|\sigma_{n} f(0)\right|
$$

But $f(0)=0 \Rightarrow\left|\sigma_{n} f(0)\right| \rightarrow 0$ as $n \rightarrow \infty$. Therefore $\left\|e_{n}-f\right\|_{\infty} \rightarrow 0$, and, hence, $\left\|T_{s} e_{n}-T_{s} f\right\|_{\infty} \rightarrow 0$ as $n \rightarrow \infty$. But $T_{s} e_{n}$ is continuous for all $n$, being a polynomial of degree $n$. Thus $T_{s} f \in C$.

$(\Rightarrow)$ Take $F=f-f(0)$. Then

$$
T_{S} F(t)=T_{S} f(t)-f(0)(\operatorname{sgn} t)(\pi-|t|) / 2 \text { for a.e. } t \text {. }
$$

But $T_{S} f$ and $T_{S} F$ are continuous functions-the second one is continuous because $F(0)=0$. Hence, $f(0)=0$. Q.E.D.

Remark. Once $T_{S} f$ is continuous, it is not very hard to show that the Fourier series of $T_{S} f$ converges uniformly. We just have to consider the difference of Cesàro means and the partial sums of $T_{S} f$ and use the strong summability of Fourier series of $f$ at 0 .

COROLlaRY 1.1. Let $f$ be a continuous function and $a_{0}$ the constant term of its Fourier expansion. Then for every $x$,

$$
\lim _{t \rightarrow 0+} \frac{1}{\pi} \int_{t}^{\pi} \frac{\tilde{f}(x+u)-\tilde{f}(x-u)}{2 \tan u / 2} d u=f(x)-a_{0} .
$$

Proof. It is enough to prove this for $x=0$. We can also assume that $f=f_{c}$. Take $G=f_{c}-f_{c}(0)$. Then, by (1.1), 


$$
T_{S} G(t)=\int_{t}^{\pi} \frac{\tilde{f}_{c}(u) d u}{2 \tan u / 2}+F(t)-f_{c}(0)(\pi-t) / 2 \text { for } \pi \geqslant t>0
$$

and

$$
T_{S} G(-t)=-T_{S} G(t) \text { for } \pi \geqslant t>0 .
$$

But $T_{S} G \in C$ by the theorem. Hence,

$$
\lim _{t \rightarrow 0+} \frac{1}{\pi} \int_{t}^{\pi} \frac{\tilde{f}_{c}(u)}{2 \tan u / 2} d u=f_{c}(0) \text {. Q.E.D. }
$$

Proof of Theorem 2. $(\Leftarrow)$ Since $f$ and $T_{S} f$ are continuous by $[4$, Theorem 5], the necessity of the condition is obvious from Theorem 1.

$(\Rightarrow)$ We shall consider two cases.

Case 1. $f \in \Lambda(1, p)$ where $1<p<\infty$. By [3, Theorem 24], $f(x)-f(0)$ $=\int_{0}^{x} F(t) d t$ where $F \in L^{p}$. Let $f \sim \sum_{n=0}^{\infty}\left(a_{n} \cos n t+b_{n} \sin n t\right)$. Then

$$
F(t)=f^{\prime}(t) \sim \sum_{n=1}^{\infty}\left(-n a_{n} \sin n t+n b_{n} \cos n t\right) .
$$

So

$$
\sum_{n=1}^{\infty} n a_{n} \sin n t \sim[F(-t)-F(t)] / 2 \in L^{p} .
$$

Hence, by [1, Theorem 2],

$$
\sum_{n=1}^{\infty}\left(\sum_{j=n}^{\infty} a_{j}\right) \cos n t \sim F^{*} \in L^{p}
$$

But $\sum_{j=0}^{\infty} a_{j}=f(0)=0$. Hence

$$
-\sum_{n=1}^{\infty} S_{n}(0 ; f) \cos n t \sim F^{*} \in L^{p}
$$

Now, by [3, Theorem 22], $T_{s} f \in \Lambda(1, p)$. by

Case 2. $f \in \Lambda(\alpha, p)$ where $0<1 / p<\alpha<1$. Define $\sigma_{n} f$ as before and $\sigma_{n, j}$

$$
\sigma_{n, j}(x)=\sum_{i=0}^{j}[1-i /(n+1)] A_{i}(x ; f) .
$$

By [7, Theorem 6], $\left\|f-\sigma_{n} f\right\|_{p}=O\left(n^{-\alpha}\right)$. Now $T_{S} \sigma_{n} f=P_{n}+Q_{n}$ where

$$
P_{n}(t)=\sum_{j=1}^{n}\left[\frac{\sigma_{n j}(0)}{j}\right] \sin j t \quad \text { and } \quad Q_{n}(t) \sim\left[\sigma_{n} f(0)\right] \sum_{j=n+1}^{\infty} \frac{(\sin j t)}{j} \text {. }
$$

By [4, Theorem 5], $f \in \Lambda(\alpha-1 / p)$. Hence, by [9, Vol. I, p. 123], $\left|\sigma_{n} f(0)\right|$ $=O\left(n^{-\alpha+1 / p}\right)$. Now

$$
\sum_{j=1}^{\infty}(\sin j t) / j \sim(\operatorname{sgn} t)(\pi-|t|) / 2 \in \Lambda(1 / p, p) .
$$

Hence, by [7, Theorem 5], $\left\|Q_{n} / \sigma_{n} f(0)\right\|_{p}=O\left(n^{-1 / p}\right)$.Using Lemma 1.1 and the above relations, we get 


$$
\begin{aligned}
\left\|T_{S} f-P_{n}\right\|_{p} & \leqslant\left\|T_{S} f-T_{S} \sigma_{n} f\right\|_{p}+\left\|T_{S} \sigma_{n} f-P_{n}\right\|_{p} \\
& \leqslant A_{p}\left\|f-\sigma_{n} f\right\|_{p}+\left\|Q_{n}\right\|_{p}=O\left(n^{-\alpha}\right) .
\end{aligned}
$$

Hence, by [7, Theorem 2], $T_{s} f \in \Lambda(\alpha, p)$. Q.E.D.

REMARKS. (i) Using the above technique, we can easily show that $f \in \Lambda(\alpha)$ and $f(0)=0 \Rightarrow T_{s} f \in \Lambda(\alpha)$ where $0<\alpha<1$.

(ii) Using Corollary 2.1 of [8], we can also show that $f \in \Lambda(1,1) \Rightarrow T_{S} f \in$ $\Lambda(1,1)$. But the corresponding statement for the class $\Lambda(1 / p, p)$, where $p>1$, is not true as can be seen by taking $f \sim \sum_{n=1}^{\infty}(\cos n x) / n$. Imposing the condition $\left|\sigma_{n} f(0)\right|=O(1)$, and using the above technique, one can prove that $f \in \Lambda(1 / p, p)$ implies $T_{S} f \in \Lambda(1 / p, p)$.

\section{REFERENCES}

1. R. Bellman, Note on a theorem of Hardy on Fourier constants, Bull. Amer. Math. Soc. 50(1944), 741-744. MR6, 125.

2. G. H. Hardy, Notes on some points in the integral calculus, Messenger Math. 58(1929), 50-52.

3. G. H. Hardy and J. E. Littlewood, Some properties of fractional integrals. I, Math. Z. 27(1928), 565-606.

4. , A convergence criterion for Fourier series, Math. Z. 28(1928), 612-634.

5. M. Kinukawa and S. Igari, Transformations of conjugate functions, Tôhoku Math. J. (2) 13(1961), 274-280. MR26 \#2829.

6. A. A. Konyuškov, On Lipschitz classes, Izv. Akad. Nauk SSSR Ser. Mat. 21(1957), 423-448. (Russian) MR19, 546.

7. E. S. Quade, Trigonometric approximation in the mean, Duke Math. J. 3(1937), 529-543.

8. Rajendra Sinha, On an infinite linear combination of partial sums of Fourier series, Studia Math. (to appear).

9. A. Zygmund, Trigonometric series. Vols. I, II, 2nd ed., Cambridge Univ. Press, New York and London, 1968. MR38 \#4882.

Department of Mathematics. Purdue University, West Läisayette, Iindiana 47907

Current address: Department of Mathematics, Birla Institute of Technology and Science, Pilani, Rajasthan, India 333031 